\title{
IMPLANTABLE CARDIAC DEVICE INFECTION - A CLINICAL AUDIT
}

\author{
Muhammad Asad, Qurban Hussain Khan, Mir Waqas Baloch, Kumail Abbas Khan, Muhammad Amer Naseem, \\ Azmat Hayat, Noor Shah \\ Armed Forces Institute of Cardiology/National Institute of Heart Disease (AFIC/NIHD)/National University of Medical Sciences (NUMS) \\ Rawalpindi Pakistan
}

\section{ABSTRACT}

Objective: Cardiac Implantable Electronic Devices are being implanted more commonly now compared to the past. Due to the rise in implantation rate complication have also considered to increase. One of the dreadful complications is devices infection. This study was conducted to assess retrospectively the rate of device infection and risk factors associated with it.

Study Design: Observational study.

Place and Duration of Study: AFIC/NIHD, Rawalpindi, from Jan 2018 to Jan 2019.

Methodology: A total of 356 patients who underwent cardiac devices implantation. Their records were studied and all the patients who developed device infections were further reviewed in detail. Demographic details, clinical, laboratory data and imaging records were evaluated. Patients were classified into different categories of infections based on predefined criteria according to the guidelines. Risk factors were also taken into account.

Results: Out of 356 devices 14 got infected and infection rate was 3.9\%. Generator site infection was seen in 6 followed by generator erosion in 5 while 2 had pocket site infection with bacteremia and 1 developed pocket site infection with lead/valvular endocarditis. Dual chamber permanent pacemakers were infected the most. Denovo devices had high infection rate compared to replacement. Microbes were identified in 3 patients.

Conclusions: Our findings suggest that the increasing incidence of Cardiac Implantable electronic devices infection in current clinical settings was multifactorial. Care should be taken at every step starting from preoperative, intraoperative to postoperative stage for prevention of device infection.

Keywords: Cardiac implantable devices, Infection, Incidence.

This is an Open Access article distributed under the terms of the Creative Commons Attribution License (http://creativecommons.org/licenses/by/4.0), which permits unrestricted use, distribution, and reproduction in any medium, provided the original work is properly cited.

\section{INTRODUCTION}

Cardiac Implantable Electronic Devices (CIED) including permanent pacemakers (PPMs), implantable cardioverter-defibrillators (ICDs) and cardiac resynchronization therapy (CRT-D [with defibrillator] and CRT-P [without defibrillator] are being implanted for the past few decades and the numbers are expected to increase in future due to increased life expectancy of the patients as well as population growth ${ }^{1}$. The implantation of these devices has resulted in a significant improvement in both patient quality of life and longevity. Moreover there has also been a rise in device revisions, upgrades and replacements ${ }^{2}$.

Due to increase in the procedural numbers

Correspondence: Dr Muhammad Asad, EP Department, AFIC/ NIHD Rawalpindi Pakistan the rate of complications is also considered to have increased. One of the most serious complications is device infection carrying a high morbidity and mortality and also results in a significant cost burden to health care system. Reasons for this trend are uncertain, but likely relate to increasing proportions of implantable cardioverter defibrillator (ICD) and cardiac res-ynchronization therapy (CRT) devices implanted, as well as implantations in 'higher risk' patients, i.e. patients with diabetes, heart failure and renal failure ${ }^{3}$. CIED infections frequently require removal of both leads and generator along with prolonged intravenous antibiotic therapy. CIED infection rates vary significantly between studies but are commonly reported to be between $1 \%$ and $7 \%$ associated with high fatality that ranges from $3 \%$ $19 \%$ of patients 4 . Prutkin et al, reported that a total of $3390(1.7 \%)$ out of 200,909 implanted ICDs developed device infection within six months 5 . 
In literature risk factors which have been considered to predispose patients to infection include presence of other comorbidities like coexisting organs malfunction, number of implanted leads, female group and use of steroids. Multiple leads and device revision or replacement are also identified as independent markers of device infection 6 .

In majority (88\%) of cases infections are caused by Gram-positive organisms commonest among them was found to be methicillin sensitive Staphylococcus aureus (MSSA; 30.8\%) followed by coagulase negative group including Staphylococcus (20.5\%). Methicillin-resistant Staph aureus (MRSA) is found in half of the cases ${ }^{7}$.

CIED infections not only cause physical and psychological stress to patients but also have financial implications to the healthcare system which include readmission, prolonged stay in hospital, and cost of removal of device and leads, insertion of a new device, diagnostic workups and medical therapy for infection ${ }^{8}$. Our objective was to study the incidence of device infections in our hospital and associated mortality.

\section{METHODOLOGY}

The study was performed at a 400-bed academic tertiary care medical center located in Rawalpindi, Pakistan. All the patients implanted with CIED between Jan 2018 to Jan 2019 were included in the audit. Preoperative data collected included patient demographic data, medical comorbidities and any antiplatelet or anticoagulant being used, preoperative hair removal when necessary, skin preparation with chlorhexidine gluconate $(\mathrm{CHG})$ and povidone-iodine, and preoperative antimicrobial prophylaxis. Procedural data collected included the type of CIED implanted (pacemaker or defibrillator), number of leads placed and initial placement versus revision/ replacement.

Clinical definition of infection was used to identify the presence and extent of infection. It was divided into following categories namely 1) Isolated generator pocket infection defined as localized erythema, swelling, pain, tenderness, warmth, or drainage with negative blood culture 2) Isolated pocket erosion: device and/or lead (s) are through the skin, with exposure of the generator or leads, with or without local signs of infection 3) Pocket site infection with bacteremia: local infection signs and positive blood cultures 4) Lead infection: lead vegetation and positive blood cultures 5) Pocket site infection with lead/ valvular endocarditis: localsigns and positive blood cultures and lead or valvular vegetation (s) 6) CIED endocarditis without pocket infection: positive blood cultures and lead or valvular vegetation (s).

Modified duke criteria were used to confirm the presence of positive blood cultures along with valvular or lead vegetation on echocardiography. Bacterial cultures were used to confirm both types of CIED infections by positive cultures from the device $\backslash$ pocket, extracted leads or blood.

\section{RESULTS}

The total number of implantable cardiac devices in one year duration (2018-2019) was 356 out of which PPM single chamber were 23 (6.4\%), 285 (80.05\%) PPM dual chamber, 15 (4.2\%) were ICD single chamber while 11 (3.08\%) were ICD dual chamber. Six (1.68\%) were CRTD and CRTP were also $6(1.68 \%)$. Among 356 devices there were $333(93.5 \%)$ new implants and 23 (6.2\%) were replacement procedures. In our study population 257 (72.19\%) were males while 99 (27.8\%) were females. The mean age of the population was $66.53 \pm 15.28$ years. Risk factors were diabetes mellitus in 100 (28\%) patients, Hypertension $50(14 \%)$, Heart failure $18(0.5 \%)$, Chronic renal failure $20(0.5 \%)$, antiplatelet $3(0.08 \%)$, anticoagulant $1(0.02 \%)$, malignancy $2(0.05 \%)$ respectively as shown in table-I.

There were 14 device infections which makes up infection rate $3.9 \%$. Ppm dual chamber denovo implantation were the ones most commonly infected $10(71.4 \%)$, followed by dual chamber replacement $2(1.4 \%)$ de-novo single chamber 1 $(0.7 \%)$ and denovo single chamber ICD 1 (0.7\%). Six $(42.8 \%)$ patients had isolated generator pocket infection, $5(35.7 \%)$ had isolated generator ero- 
sion, $2(1.4 \%)$ patient had a pocket site infection with bacteremia, and $1(0.7 \%)$ patient hadpocket

Table-I: Clinical characteristics and and device data of the patients.

\begin{tabular}{|c|c|}
\hline $\begin{array}{l}\text { Demographic } \\
\text { Characteristics }\end{array}$ & $\begin{array}{c}\text { Mean } \pm \text { SD } \\
/ \mathrm{n}(\%)\end{array}$ \\
\hline Age & $66.53 \pm 15.28$ \\
\hline \multicolumn{2}{|l|}{ Gender } \\
\hline Male & $257(72.19 \%)$ \\
\hline Female & $99(27.8 \%)$ \\
\hline \multicolumn{2}{|l|}{ Co-morbids } \\
\hline Hypertension & $50(14.04 \%)$ \\
\hline Diabetes Mellitus & $100(28.08 \%)$ \\
\hline Heart failure & $18(0.5 \%)$ \\
\hline Chronic renal failure & $20(0.5 \%)$ \\
\hline Antiplatelet & $3(0.08 \%)$ \\
\hline Anticoagulant & $1(0.02 \%)$ \\
\hline Malignancy & $2(0.05 \%)$ \\
\hline \multicolumn{2}{|l|}{ Pre op parameters } \\
\hline Prophylactic antibiotic & $356(100 \%)$ \\
\hline Hair clipping & $354(98.3 \%)$ \\
\hline Skin preparation & $356(100 \%)$ \\
\hline \multicolumn{2}{|c|}{ Type of Device Implanted } \\
\hline PPM Single chamber & $23(6.4 \%)$ \\
\hline PPM Dual chamber & $285(80.5 \%)$ \\
\hline ICD Single chamber & $15(4.2 \%)$ \\
\hline ICD Dual chamber & $11(3.02 \%)$ \\
\hline CRTD & $6(1.68 \%)$ \\
\hline CRTP & $6(1.68 \%)$ \\
\hline \multicolumn{2}{|l|}{ Type of Implantation } \\
\hline De-novo & $333(93.5 \%)$ \\
\hline Replacement & $23(6.5 \%)$ \\
\hline
\end{tabular}

\section{DISCUSSION}

In this study $3.9 \%$ patients developed infection which was slightly higher than the guidelines accepted infection rate $(0.2-3.7 \%)^{9}$. Operations were performed by either consultant electrophysiologist or fellow electrophysiology under supervision; hair clipping was done in all but 2 patients according to $\mathrm{CDC}$ recommendations and skin preparation was done first with povidone iodine for 2-3 days and then with combination of povidone iodine and chlorhexidine gluconate (CHG) on the operating table just before the procedure $^{10}$. Studies have shown that repeated skin cleansing is considered more effective than single intense scrubbing as It allows the disinfectant to travel to deeper layers of the skin and disinfect hair follicles as well11,12. Perioperative prophylactic antibiotic cefoperazone $1 \mathrm{~g}$ hour before the procedure was given in 338 (95\%) patients while $18(5 \%)$ were given vancomycin due to cephalosporin allergy. This practice has been supported by a number of studies ${ }^{13,14}$.

Various factors described in studies have been reported to predispose to PPM/ICD. These vary from either being patient centered or linked to procedural techniques or device related ${ }^{15}$. A meta-analysis from pooled anaysis comprising of 206,176 patients showed that Esrd, Copd, diabetes, fever were commonly seen among patients who developed infection while duration, pocket

Table-II: Infected cases data.

\begin{tabular}{l|l|l|c}
\hline \multirow{2}{*}{ Devices } & & De-novo dual chamber & 10 \\
& Permanent Pacemakers ICD & De-novo single chamber & Replacement (dual chamber) \\
& & De-novo single chamber & 1 \\
\hline \\
Types of & Isolated generator pocket infection & & 1 \\
CIED & Isolated generator erosion & & 5 \\
& Pocket site infection with bacteremia & & 2 \\
& Pocket site infection with lead/valvular & & 1 \\
\hline
\end{tabular}

site infection with lead/valvular endocarditis. Microbes were identified in 3 cases only and they included staphylococcus aureus, pseudomonas aeruginosa and staphylococcus epidermidis as shown in table-II. hematoma, inexperienced operator, temoprary pacing wire, abdominal location, epicardial leads were some of the procedure and device related risk factors ${ }^{16}$. We observed that males appear to be at higher risk of infection than females. The 
median age did not differ significantly between infected and non-infected cases. Patients implanted with de-novo dual chamber devices were at higher risk and common risk factors seen in our patients were diabetes, hypertension, heart failure and chronic renal failure. Isolated generator pocket infection $6(42.8 \%)$ and $5(35.7 \%)$ isolated generator erosion were the commonest presentation of CIED infections in our study population. $10(71.4 \%)$ of the cases presented within 6 months of the index procedure while $4(28.5 \%)$ of them were infected after 14 months of device implantation. Three $(60 \%)$ patients with generator erosion had low normal BMI (mean $19 \mathrm{~kg} / \mathrm{m}^{2}$ ) and 1 (20\%) patient was on steroid. Four $(66.6 \%)$ out of 6 patients of isolated pocket infection were preceded by skin boil which were managed conservatively at another hospital.

Fever was present in $10(71.4 \%)$ of cases and average temperature was $39.7^{\circ} \mathrm{C}$; all the patients diagnosed as isolated generator pocket infection, pocket site infection with bacteremia, pocket site infection with lead/valvular endocarditis had fever while only 1 patient with generator erosion developed fever.

Blood cultures were sent in $12(85.7 \%)$ of cases and swab from pocket for culture and sensitivity was sent in all. All but $2(85.7 \%)$ cases samples were drawn before any antibiotic was given although guidelines recommend obtaining cultures prior to the institution of antibiotic therapy $^{15}$. No growth was seen in any of the pocket swabs but microbes were identified in $3(21.4 \%)$ of the cases from blood samples. These included staphylococcus aureus, pseudomonas aeruginosa and staphylococcus epidermidis. In these patients samples were drawn before any antibiotic was given.

Transthoracic echocardiography (TTE) was performed in all the patients diagnosed as infection while 1 patient who was suspected infective endocarditis underwent transesophageal echocardiography (TOE). Lead vegetations were found in 1 patient and vegetations were located on right atrial and ventricular leads sparing the valves.
Guidelines ${ }^{17}$ suggest doing TOE in all patients who have documented or suspected bloodstream infection or CIED pocket infection but due to undocumented reasons TEE could not be done in our patients.

Regarding management all patients with CIED patients were treated with antibiotics as per recommendations and also considered for removal of generator as well as leads. Antibiotics were given for minimum 6 weeks. Generators were explanted in all infected cases but lead extraction was possible in 10 cases $(71 \%)$. Reimplantation was done on the contralateral infraclavicular region after the wound healed along with the waiting period of minimum 7 days with optimal antibiotic therapy as per the recommendations 18 . Those patients whose leads could not be removed were offered surgical opinion but all patients refused to undergo procedure so they were kept on long term antibiotic therapy.

An infection-related mortality rate at our institution was $2.1 \%$. Two patients were lost to follow up however the remainder remain infection free in visits after 1 month and 3 months so true relapse could not be known.

\section{RECOMMENDATIONS}

Although this audit looked at some but not all attributes related to the CIED infections, however, recommendations can be given to decrease the infection rate. Pre-operative site where device is being implanted should be assessed thoroughly and hair should be clipped in all patients when required, diabetic control should be optimized before implantation. Moreover temporary pacing wire should not stay long and efforts should be done to implant permanent pacemaker as early as possible.

Those patients who develop superficial infections over the generator site should be followed up closely. Blood cultures should be sent before first dose of antibiotic is given when infection is suspected. Antibiotics may be considered in those patients who present with skin erosion even if fever is not present. 
Since the resources are limited as in our part of the world and most of patients cannot afford costly procedures of lead explants like laser lead extraction so patient education may play an important role along with regular follow ups to the hospital in preventing infections. Nevertheless future studies are required before any local guidelines can be developed.

\section{CONFLICT OF INTEREST}

This study has no conflict of interest to be declared by any author.

\section{REFERENCES}

1. Baddour LM, Epstein AE, Erickson CC, Knight BP, Levison ME, Lockhart PB, et al.Update on cardiovascular implantable electronic device infections and their management: a scientific statement from the American Heart Association.Circulation 2010; 121(3): 458-77.

2. Kurtz SM, Ochoa JA, Lau E, Shkolnikov Y, Pavri BB, Frisch D, et al. Implantation trends and patient profiles for pacemakers and implantable cardioverter defibrillators in the United States: 1993-2006. Pacing Clin Electrophysiol 2010; 33(6): 705-11.

3. Johansen JB, Jørgensen OD, Møller M, Arnsbo P, Mortensen PT, Nielsen JC. Infection after pacemaker implantation: infection rates and risk factors associated with infection in a populationbased cohort study of 46299 consecutive patients. Eur Heart J 2011; 32(8): 991-8.

4. Lai KK, Fontecchio SA. Infections associated with implantable cardioverter defibrillators placed transvenously and via thoracotomies: epidemiology, infection control, and management. Clin Infect Dis 1998; 27(2): 265-69.

5. Prutkin JM, Reynolds MR, Bao H, Curtis JP, Al-Khatib SM, Aggarwal S, et al. Rates of and factors associated with infection in 200909 Medicare implantable cardioverter-defibrillator implants: results from the National Cardiovascular Data Registry. Circulation 2014; 130(13): 1037-43.

6. Tarakji KG, Ellis CR, Defaye P, Kennergren C. Cardiac implantable electronic device infection in patients at risk. Arrhythmia \& electrophysiology review. 2016; 5(1): 65-71.

7. Margey R, McCann H, Blake G. Contemporary management of and outcomes from cardiac device related infections. Europace 2010; 12(1): 64-70.
8. Ng CY, Mela T. A Primer on Cardiac Devices: Psychological and Pharmacological Considerations. Psychiatric Annals. 2016 ; 46(12): 683-90.

9. Persson R, Earley A, Garlitski AC, Balk EM, Uhlig K. Adverse events following implantable cardioverter defibrillator implantation: a systematic review. J Interv Card Electrophysiol 2014; 40(2): 191-205.

10. Mangram J, Horan TC, Pearson ML, Silver LC, Jarvis WR. "Guideline for prevention of surgical site infection," Infection Control \& Hospital Epidemiol 1999; 20(4): pp.247-78.

11. Sistla SC, Prabhu G, Sistla S, Sadasivan J. Minimizing wound contamination in a 'clean surgery: comparison of chlorhexidineethanol and povidoneiodine. Chemother 2010; 56(4): 261-67.

12. Paocharoen V, Mingmalairak C, Apisarnthanarak A. Comparison of surgical wound infection after preoperative skin preparation with $4 \%$ chlorhexidine and povidone iodine: a prospective randomized trial. J Med Associat Thail 2009; 92(7): 898-02.

13. Nishioka SA, Varejão T, Uipe D, Pedrosa AA. Efficacy of antibiotic prophylaxis before the implantation of pacemakers and cardioverter-defibrillators: results of a large, prospective, randomized, doubleblinded, placebo-controlled trial. Circ Arrhythm Electrophysiol 2009; 2(1): 29-34.

14. Sohail MR, Uslan DZ, Khan AH, Friedman PA, Hayes DL, Wilson WR, et al. Risk factor analysis of permanent pacemaker infection. Clin Infect Dis 2007; 45(2): 166-73.

15. Blomström-Lundqvist $C$, Traykov V, Erba PA, Burri H, Nielsen JC, Bongiorni MG, et al. European Heart Rhythm Association (EHRA) international consensus document on how to prevent, diagnose, and treat cardiac implantable electronic device infections-endorsed by the Heart Rhythm Society (HRS), the Asia pacific heart rhythm society (APHRS), the Latin American Heart Rhythm Society (LAHRS), international society for cardiovascular infectious diseases (ISCVID) and the european society of clinical microbiology and infectious diseases (ESCMID) in collaboration with the European Association for EP. Europace 2020; 22(4): 515-49.

16. Polyzos KA, Konstantelias AA, Falagas ME. Risk factors for cardiac implantable electronic device infection: a systematic review and meta-analysis. Europace 2015; 17(1): 767-77.

17. Klug D, Lacroix D, Savoye C, Goullard L, Grandmougin D, Hennequin JL, et al. Systemic infection related to endocarditis on pacemaker leads: clinical presentation and management. Circulation 1997; 95(8): 2098-07.

18. Kusumoto FM, Schoenfeld MH, Wilkoff BL, Berul CI, Birgersdotter-Green UM. 2017 HRS expert consensus statement on cardiovascular implantable electronic device lead management and extraction. Heart Rhythm 2017; 14(1): e503-51. 